Pacific Journal of Mathematics

MOSAICS OF METRIC CONTINUA AND OF QUASI-PEANO 


\title{
MOSAICS OF METRIC CONTINUA AND OF QUASI-PEANS SPACES
}

\author{
LORRAINE D. LAVALLEE
}

1. Introduction. A collection $\left\{\left(X_{a}, \mathscr{T}_{a}\right): a \in A\right\}$ is a mosaic of topological spaces on a set $X$ if and only if each $\left(X_{a}, \mathscr{T}_{a}\right)$ is a topological space; $X=\cup\left\{X_{a}: a \in A\right\}$; and the following compatibility condition is satisfied: for all $a, b \in A$ and all subsets $M$ of $X_{a}$, if $M$ is $\mathscr{T}_{a}$-closed then $M \cap X_{b}$ is $\mathscr{T}_{b}$-closed. For a mosaic of topological spaces on $X$, the mosaic topology $\mathscr{T}$ is defined as follows: for all $M \cong X, M$ is $\mathscr{T}$-closed if and only if $M \cap X_{a}$ is $\mathscr{T}_{a}$-closed for all $a \in A$. Clearly, each $\left(X_{a}, \mathscr{T}_{a}\right)$ is then a closed subspace of $(X, \mathscr{T})$. If each $\left(X_{a}, \mathscr{T}_{a}\right)$ is a compact metric space, a Peano space, or an arc then $(X, \mathscr{T})$ is called a mosaic space, a curve space, or an arc space, respectively.

Davison [1] introduced the theory of mosaics of topological spaces, concentrating on the theory of mosaic spaces and establishing some properties of curve spaces and arc spaces. Our purpose is to study mosaics of spaces which are between being compact metric and Peano; namely, compact, connected, metric spaces and compact, locally connected, metric spaces, which we shall call metric continua and quasiPeano spaces, respectively. Mosaics of these spaces with the mosaic topology will be called m-continuum spaces and quasi-curve spaces, respectively.

It might seem quite natural to consider, also, mosaics of compact metric spaces each of which has only finitely many components. Doing so, however, yields nothing more than is obtained by studying mosaics of metric continua, as Theorem 3.3 will show.

In this paper, we give a characterization of $m$-continuum spaces and sufficient conditions for a mosaic spaces to be an $m$-continuum space. The property of $m$-strong connectedness is studied in connection with $m$-continuum spaces and a sufficient condition for an $m$-continuum space to be locally $m$-strongly connected is given. Finally, the equivalence of curve spaces and quasi-curve spaces is established.

For ease of reference we now list results of Davison to be used in this paper.

1.1. Notation. If $S$ is a sequence of points, $S_{J}$ denotes the point set associated with $S$.

1.2. TheOREM. ([2], Lemma 1.5) Let $(X, \mathscr{T})$ be a mosaic space

Received January 29,1963 , and in revised form October 20,1963. This material is based on a portion of a dissertation submitted to the Graduate School of the University of Michigan. 
eetermined by a mosaic $\left\{\left(X_{a}, \mathscr{T}_{a}\right): a \in A\right\}$ of compact metric spaces. If a sequence $S$ in $X \mathscr{T}$-converges to $x \in X$, then there exists a subsequence $S^{\prime}$ of $S$ and an $a \in A$ such that $S_{J}^{\prime} \cup\{x\} \cong X_{a}$.

\subsection{LemMa. ([2], p. 527) A mosaic space is a $T_{1}$-space.}

1.4. THEOREM. ([2], Corollary 1.9) If $(X, \mathscr{T})$ is a mosaic space, every $\mathscr{T}$-compact subset is $\mathscr{T}$-closed.

1.5. Definition. ([2], p. 526) A topology $\mathscr{T}$ on a set $X$ is full if and only if the following statement holds: for all $M \subseteq X$, if $M$ is not $\mathscr{T}$-closed then there exists a sequence $S$ and a point $x \in X$ such that $S_{J} \subseteq M, x \notin M$ and $S \mathscr{T}$-converges to $x$.

1.6. Theorem. ([2], Theorems 1.3. and 1.6, Corollary 2.3.) $A$ space $(X, \mathscr{T})$ is a mosaic space if and only if $\mathscr{T}$ is full and limits of $\mathscr{T}$-convergent sequences are unique.

1.7. THEOREM. ([2], Theorem 4.1.) Every open or closed subspace of a mosaic space is a mosaic space.

2. A characterization of m-continuum spaces.

2.1. Theorem. A space $(X, \mathscr{T})$ is an m-continuum space if and only if the following three conditions hold:

(i) $\mathscr{T}$ is full;

(ii) limits of $\mathscr{T}$-convergent sequences are unique;

(iii) for every sequence $S$ and every point $x$ in $X$, if $S \mathscr{T}$-converges to $x$ then there exists a subsequence $S^{\prime}$ of $S$ contained in some metric continuum in $X$.

Proof. If $(X, \mathscr{T})$ is an $m$-continuum space, then properties (i), (ii) and (iii) follow from Theorems 1.6 and 1.2.

Conversely, suppose $(X, \mathscr{T})$ is a space satisfying conditions (i), (ii) and (iii). Let $\left\{\left(X_{a}, \mathscr{T}_{a}\right): a \in A\right\}$ be the collection of all metric continua in $(X, \mathscr{T}) .(X, \mathscr{T})$ is a mosaic space by Theorem 1.6. Theorem 1.4 then implies that $\left\{\left(X_{a}, \mathscr{T}_{a}\right): a \in A\right\}$ is a mosaic of metric continua. Let $\mathscr{T}^{\prime}$ be the $m$-continuum topology for this mosaic of metric continua on $X$. If $F$ is a $\mathscr{T}$-closed subset of $X$ then $F \cap X_{a}$ is $\mathscr{T}$-closed and so $\mathscr{T}_{a}$-closed for all $a \in A$. Hence $F$ is $\mathscr{T}^{\prime}$-closed and it follows that $\mathscr{T} \cong \mathscr{T}^{\prime}$. Conversely, if $F$ is $\mathscr{T}^{\prime}$-closed then $F \cap X_{a}$ is $\mathscr{T}_{a}$-closed for all $a \in A$. Now let $S$ be a sequence such that $S \mathscr{T}$-converges to $x \in X$ and $S_{J} \subseteq F$. By (iii) there exists a subsequence $S^{\prime}$ of $S$ and an $a \in A$ such that $S_{J}^{\prime} \cup\{x\} \subseteq X_{a}$. Furthermore, $S^{\prime} \mathscr{T}_{a}$-converges to $x$. 
Since $S_{J}^{\prime} \subseteq F \cap X_{a}$ and $F \cap X_{a}$ is $\mathscr{T}_{a}$-closed, then $x \in F \cap X_{a} \subseteq F$. Since $\mathscr{T}$ is full and $F$ contains all its sequential limit points then $F$ is $\mathscr{T}$-closed and it follows that $\mathscr{T}^{\prime} \cong \mathscr{T}$. Thus $\mathscr{T}^{\prime}=\mathscr{T}$ and $(X, \mathscr{T})$ is an $m$-continuum space.

As the proof of Theorem 2.1 shows, any space $(X, \mathscr{T})$ which satisfies the three conditions of this theorem is that $m$-continuum space which is determined by the mosaic of all its metric subcontinua.

2.2. CoRollary. Every locally compact, locally connected, mosaic space $(X, \mathscr{T})$ is an m-continuum space.

Proof. By Theorem 1.6, conditions (i) and (ii) of Theorem 2.1 are satisfied. Let $S$ be any sequence in $X$ which $\mathscr{T}$-converges to $x \in X$. If $U$ is any $\mathscr{T}$-open set containing $x$, the local compactness of $(X, \mathscr{T})$ implies the existence of a $\mathscr{T}$-open set $V$ such that $x \in V \subseteq \bar{V} \subseteq U$ and $\bar{V}$ is compact. Let $C$ be the component of $x$ in $V$. Since $(X, \mathscr{T})$ is locally connected, $C$ is $\mathscr{T}$-open. Thus there exists a subsequence $S^{\prime}$ of $S$ such that $S_{J}^{\prime} \cup\{x\} \subseteq C$. Since $C \cong \bar{C}$ and $\bar{C}$ can easily be seen to be a metric continuum, condition (iii) of Theorem 2.1 is now satisfied. Hence $(X, \mathscr{T})$ is an $m$-continuum space.

3. A sufficient condition for a mosaic space to be an $m$-continuum space.

3.1. THEOREM. Every component of an m-continuum space is open.

Proof. Let $(X, \mathscr{T})$ be an $m$-continuum space determined by a mosaic $\left\{\left(X_{a}, \mathscr{T}_{a}\right): a \in A\right\}$ of metric continua and let $C$ be a component of $(X, \mathscr{T})$. For each $a \in A$ either $C \cap X_{a}=\phi$ or $C \cap X_{a}=X_{a}$. Hence $C \cap X_{a}$ is $\mathscr{T}_{a}$-open for all $a \in A$ and so $C$ is $\mathscr{T}$-open.

Of course, an $m$-continuum space need not be locally connected; in fact, every metric continuum is trivially an $m$-continuum space.

3.2. Lemma. If $(X, \mathscr{T})$ is a compact space in which components are open, then the number of components in $(X, \mathscr{T})$ is finite.

3.3. Theorem. Let $(X, \mathscr{T})$ be a mosaic space described by a mosaic $\left\{\left(X_{a}, \mathscr{T}_{a}\right): a \in A\right\}$ of compact metric spaces. If, for each $a \in A$, the components of $\left(X_{a}, \mathscr{T}_{a}\right)$ are $\mathscr{T}_{a}$-open, then $(X, \mathscr{T})$ is an m-continuum space. [In fact, $(X, \mathscr{T})$ is that m-continuum space which is described by the mosaic of all components of all the $\left(X_{a}, \mathscr{T}_{a}\right)$, $a \in A]$. 
Proof. For each $a \in A$, let $\left\{\left(X_{a p}, \mathscr{T}_{a p}\right): p \in P_{a}\right\}$ be the collection of all components of $\left(X_{a}, \mathscr{T}_{a}\right)$. Each $\left(X_{a p}, \mathscr{T}_{a p}\right)$ is clearly a metric continuum. By the hypothesis and by Lemma 3.2, $P_{a}$ is finite for all $a \in A$. It is easy to see that $\left\{\left(X_{a p}, \mathscr{T}_{a p}\right): a \in A, p \in P_{a}\right\}$ is a mosaic of metric continua; let $\mathscr{T}^{\prime}$ be the topology it defines on $X$. We show that $\mathscr{T}^{\prime}=\mathscr{T}$. Suppose $F$ is any $\mathscr{T}$-closed subset of $X$. Then $F \cap X_{a}$ is $\mathscr{T}_{a}$-closed for all $a \in A$ and so $F \cap X_{a p}=\left(F \cap X_{a}\right) \cap X_{a p}$ is $\mathscr{T}_{a p}$ closed for all $a \in A$ and $p \in P_{a}$. Thus $F$ is $\mathscr{T}^{\prime}$-closed and $\mathscr{T} \cong \mathscr{T}^{\prime}$. Now suppose $F$ is a $\mathscr{T}^{\prime}$-closed subset of $X$. Then $F \cap X_{a p}$ is $\mathscr{T}_{a p}$ closed for all $a \in A$ and $p \in P_{a}$. Consequently the finiteness of each indexing set $P_{a}$ implies that $F \cap X_{a}=\cup\left\{F \cap X_{a p}: p \in P_{a}\right\}$ is $\mathscr{T}_{a}$-closed for each $a \in A$. Thus $F$ is $\mathscr{T}$-closed and $\mathscr{T}^{\prime} \subseteq \mathscr{T}$. Since $\mathscr{T}=\mathscr{T}^{\prime}$, $(X, \mathscr{T})$ is an $m$-continuum space.

The converse of Theorem 3.3 is clearly false. For instance, the plane with its usual topology is an $m$-continunm space by Theorem 2.1, but it can also be described as the mosaic of all its compact metric subspaces and these include spaces in which not all components are open.

The next example shows that Theorem 3.3 need not hold if we require only that the components of the mosaic space $(X, \mathscr{T})$ be open.

3.4. Example. ([6], Example 2, p. 84) Let $X$ be the subset of the plane defined by $X=\{(x, y):-\infty<x<+\infty, y=1\} \cup\{(x, y)$ : $-\infty<x<+\infty, y=0\} \cup\{(x, y):-\infty<x<+\infty, y=-1\} \cup\left\{R_{n}: n=\right.$ $1,2, \cdots\}$, where $R_{n}$ is a rectangle with center at $(0,0)$ and sides of length $2 n$ and $2-1 / n$ parallel to the $x$-axis and $y$-axis, respectively; let $\mathscr{T}$ be the usual metric topology on $X .(X, \mathscr{T})$ is a connected mosaic space. However, $(X, \mathscr{T})$ is not an $m$-continuum space since the sequence $S$, for which $S_{J}=\{(0,(2 n-1) / 2 n): n=1,2, \cdots\}, \mathscr{T}$ converges to $(0,1)$ and there exists no subsequence $S^{\prime}$ of $S$ such that $S_{J}^{\prime} \cup\{(0,1)\}$ is contained in a metric continuum in $(X, \mathscr{T})$.

4. The equivalence of $m$-constituents and components in an $m$-continuum space.

4.1. Definition. We shall say that the points $x$ and $y$ of a space $(X, \mathscr{T})$ are $m$-strongly connected in $(X, \mathscr{T})$ if and only if there exists a metric continuum in $(X, \mathscr{T})$ which contains $x$ and $y .(X, \mathscr{T})$ will be said to be $m$-strongly connected if and only if every two points of $(X, \mathscr{T})$ are $m$-strongly connected.

Being $m$-strongly connected is an equivalence relation and the equivalence sets into which the elements of the space are divided will be called $m$-constituents; $m$-constituents need be neither open nor closed. 
4.2. TheOREM. Let $(X, \mathscr{T})$ be a mosaic space determined by a mosaic $\left\{\left(X_{a}, \mathscr{T}_{a}\right): a \in A\right\}$ of compact metric spaces. If, for each $a \in A$, the m-constituents of $\left(X_{a}, \mathscr{T}_{a}\right)$ are $\mathscr{T}_{a}$-open then $(X, \mathscr{T})$ is an $m$ continuum space.

Proof. For each $a \in A$, the components of $\left(X_{a}, \mathscr{T}_{a}\right)$ are $\mathscr{T}_{a}$-open since they are unions of $\mathscr{T}_{a}$-open $m$-constituents. The result now follows from Theorem 3.3.

Since the converse of Theorem 3.3 fails, so does the converse of Theorem 4.2. Also, one can easily show that Theorem 4.2 fails if we require only that the $m$-constituents of $(X, \mathscr{T})$ be open; (see, for example, [4], figure $3-17$, p. 124).

4.3. THEOREM. The components of an m-continuum space are m-strongly connected.

Proof. Let $(X, \mathscr{T})$ be an $m$-continuum space determined by a mosaic $\left\{\left(X_{a}, \mathscr{T}_{a}\right): a \in A\right\}$ of metric continua, and let $C$ be a component of $(X, \mathscr{T})$. From the given mosaic define a relation $R$ on $C$ as follows: for all $x, y \in C, x R y$ if and only if there exists a finite number of metric continua $X_{a_{1}}, X_{a_{2}}, \cdots, X_{a_{n}}$ in $C$ such that $x \in X_{a_{1}}, y \in X_{a_{n}}$ and, for all $m=1,2, \cdots, n-1, \quad X_{a_{m}} \cap X_{a_{m+1}} \neq \phi . \quad R$ is an equivalence relation which partitions $C$ into disjoint subsets. Suppose there is more than one equivalence class, so that there is a set $C_{y}=\{x: x \in C$ and $x R y\}$ such that $C_{y} \neq C$. Then $C_{y} \cap X_{a}=\phi$ or $C_{y} \cap X_{a}=X_{a}$ for all $a \in A$, and so $C_{y}$ is both $\mathscr{T}$-open and $\mathscr{T}$-closed, contradicting the connectedness of $C$. Thus there is only one equivalence class and for all $x, y \in C$ there exists a finite chain of metric continua $X_{a_{1}}, \cdots, X_{a_{n}}$ in $C$ such that $x \in X_{a_{1}}, y \in X_{a_{n}}$ and, for all $m=1,2, \cdots, n-1, X_{a_{m}} \cap X_{a_{m+1}} \neq \phi$. Now $Y=\cup\left\{X_{a_{m}}: m=1,2, \cdots, n\right\}$ is clearly a compact, connected subset of $C$ which contains $x$ and $y$. By Theorem 1.4, $Y$ is $\mathscr{T}$-closed. Thus the subspace $(Y, r-\mathscr{T})$, where $r-\mathscr{T}$ is the topology $\mathscr{T}$ relativized to $y$, is a mosaic space by Theorem 1.7 and it is a $T_{1}$-space by Lemma 1.3. That $(Y, r-\mathscr{T})$ is a metric space now follows from Theorem 1.4 and from Theorem 2 in [5] that a $T_{1}$-space which is the union of a (locally) finite system of closed, metrizable subspaces is metrizable. Hence $Y=\cup\left\{X_{a_{m}}: m=1,2, \cdots, n\right\}$ is a metric continuum in $C$ which contains $x$ and $y$, and so $C$ is $m$-strongly connected.

4.4. COROLlaRY. In an m-continuum space, m-constituents and components are identical.

We remark at this point that, as the proof shows, "component" could be replaced by " $m$-constituent" in Theorem 3.1. However, this 
apparent sharpening of the result says no more in view of Corallary 4.4.

4.5. COROLlaRY. In an m-continuum space, m-constituents are both open and closed.

5. A sufficient condition for an $m$-continuum space to be locally $m$-strongly connected.

5.1. Definition. A space $(X, \mathscr{T})$ is locally $m$-strongly connected at a point $x \in X$ if and only if every open set containing $x$ contains an open, $m$-strongly connected set containing $x .(X, \mathscr{T})$ is locally $m$-strongly connected if and only if it is locally $m$-strongly connected at each of its points.

It is easy to give an example of a plane continuum $X$ which has an open subspace $Y$ such that both $Y$ and $\bar{Y}$ violate Theorem 2.1.

5.2. THEOREM. Let $(X, \mathscr{T})$ be an m-continuum space. If every open subspace of $(X, \mathscr{T})$ is an m-continum space, then $(X, \mathscr{T})$ is locally m-strongly connected.

Proof. Let $x \in X$ and let $M$ be any open subset of $(X, \mathscr{T})$ which contains $x$. By hypothesis, $(M, r-\mathscr{T})$ is an $m$-continuum space. Hence the component $C$ of $M$ which contains $x$ is both $(r-\mathscr{T})$-open and $(r-\mathscr{T})-m$-strongly connected by Theorems 3.1 and 4.3. Since $M$ is $\mathscr{T}$-open, $C$ is $\mathscr{T}$-open. Moreover, $C$ is $\mathscr{T}$ - $m$-strongly connected. Hence $(X, \mathscr{T})$ is locally $m$-strongly connected.

6. The equivalence of quasi-curve spaces and curve spaces. The main result to be obtained in this section is that quasi-curve spaces and curve spaces are equivalent. This will, of course, allow us to place less stringent conditions on the mosaic of spaces which determines a curve space. However, since Peano spaces have "nicer" properties than quasi-Peano spaces, it is more convenient to continue to work with curve spaces than with quasi-curve spaces.

6.1. Theorem. The space $(X, \mathscr{T})$ is a quasi-curve space if and only if it is a curve space.

Proof. Every curve space is clearly a quasi-curve space. Conversely, suppose $(X, \mathscr{T})$ is a quasi-curve space and that $\left\{\left(X_{a}, \mathscr{T}_{a}\right)\right.$ : $a \in A\}$ is a mosaic of quasi-Peano spaces determining $(X, \mathscr{T})$. For each $a \in A$ let $\left\{\left(X_{a p}, \mathscr{T}_{a p}\right): p \in P_{a}\right\}$ be the collection of all components of $\left(X_{a}, \mathscr{T}_{a}\right)$. For all $a \in A$ and $p \in P_{a},\left(X_{a p}, \mathscr{T}_{a p}\right)$ is a metric continuum. Since each $\left(X_{a}, \mathscr{T}_{a}\right)$ is locally connected, the components of $\left(X_{a}, \mathscr{\mathscr { T }}_{a}\right)$ 
are $\mathscr{T}_{a}$-open and so, by Theorem 3.3, $(X, \mathscr{T})$ is determined by the mosaic $\left\{\left(X_{a p}, \mathscr{T}_{a p}\right): a \in A, p \in P_{a}\right\}$ of metric continua. Since each $\left(X_{a p}, \mathscr{T}_{a p}\right), p \in P_{a}$ is $\mathscr{T}_{a}$-open and since $\left(X_{a}, \mathscr{T}_{a}\right)$ is locally connected for each $a \in A$, then each $\left(X_{a p}, \mathscr{T}_{a p}\right)$ is locally connected and is therefore a Peano space. Consequently $(X, \mathscr{T})$ is a curve space.

6.2. COROLlARY. Every quasi-curve space is an m-continuum space.

Not every $m$-continuum space is a quasi-curve space since quasicurve spaces are locally arcwise connected ([1], Theorem 4.4) whereas $m$-continuum spaces need not be even locally connected.

It would be of interest to know whether or not a locally ( $m$ strongly) connected $m$-continuum space is a quasi-curve space and whether or not the converse of Theorem 5.2 holds. These questions seem to be analogous to the as yet unanswered question posed to the author by Davison concerning whether or not a locally arcwise connected mosaic space is a quasi-curve space.

The author wishes to thank the referee for many helpful suggestions.

\section{BIBLIOGRAPHY}

1. W. F. Davison, Mosaics, thesis submitted to the University of Virginia, (1956).

2. —_, Mosaics of compact metric spaces, Trans. Am. Math. Soc., 91 (1959), 525546.

3. D. W. Hall and C. L. Spencer, Elementary Topology, Wiley, 1955.

4. J. G. Hocking and G. S. Young, Topology, Addison-Wesley, 1961.

5. J. Nagata, On a necessary and sufficient condition of metrizability, J. Inst. Polytech. Osaka City Univ., 1 (1950), 93-100.

6. M. H. A. Newman, Elements of the Topology of Plane Sets of Points, Cambridge Univ. Press, 1951.

UNIVERSITY OF MICHIGAN

AND

UNIVERSITY OF MASSACHUSETTS 



\title{
PACIFIC JOURNAL OF MATHEMATICS
}

\author{
EDITORS
}

\author{
RoBerit OsSERman \\ Stanford University \\ Stanford, California
University of Washington
Seattle 5 , Washington \\ M. G. Arsove
}

\author{
J. DugundjI \\ University of Southern California
}

Los Angeles 7, California

Lowell J. Paige

University of California

Los Angeles 24, California

\section{ASSOCIATE EDITORS}
E. F. BECKENBACH
B. H. NeumanN
F. WOLF
K. YOSIDA

\section{SUPPORTING INSTITUTIONS}

\author{
UNIVERSITY OF BRITISH COLUMBIA \\ CALIFORNIA INSTITUTE OF TECHNOLOGY \\ UNIVERSITY OF CALIFORNIA \\ MONTANA STATE UNIVERSITY \\ UNIVERSITY OF NEVADA \\ NEW MEXICO STATE UNIVERSITY \\ OREGON STATE UNIVERSITY \\ UNIVERSITY OF OREGON \\ OSAKA UNIVERSITY \\ UNIVERSITY OF SOUTHERN CALIFORNIA
}

\author{
STANFORD UNIVERSITY \\ UNIVERSITY OF TOKYO \\ UNIVERSITY OF UTAH \\ WASHINGTON STATE UNIVERSITY \\ UNIVERSITY OF WASHINGTON \\ * * * * \\ AMERICAN MATHEMATICAL SOCIETY \\ CALIFORNIA RESEARCH CORPORATION \\ SPACE TECHNOLOGY LABORATORIES \\ NAVAL ORDNANCE TEST STATION
}

Mathematical papers intended for publication in the Pacific Journal of Mathematics should by typewritten (double spaced), and on submission, must be accompanied by a separate author's résumé. Manuscripts may be sent to any one of the four editors. All other communications to the editors should be addressed to the managing editor, L. J. Paige at the University of California, Los Angeles 24, California.

50 reprints per author of each article are furnished free of charge; additional copies may be obtained at cost in multiples of 50 .

The Pacific Journal of Mathematics is published quarterly, in March, June, September, and December. Effective with Volume 13 the price per volume (4 numbers) is $\$ 18.00$; single issues, $\$ 5.00$. Special price for current issues to individual faculty members of supporting institutions and to individual members of the American Mathematical Society: $\$ 8.00$ per volume; single issues $\$ 2.50$. Back numbers are available.

Subscriptions, orders for back numbers, and changes of address should be sent to Pacific Journal of Mathematics, 103 Highland Boulevard, Berkeley 8, California.

Printed at Kokusai Bunken Insatsusha (International Academic Printing Co., Ltd.), No. 6, 2-chome, Fujimi-cho, Chiyoda-ku, Tokyo, Japan.

PUBLISHED BY PACIFIC JOURNAL OF MATHEMATICS, A NON-PROFIT CORPORATION

The Supporting Institutions listed above contribute to the cost of publication of this Journal. but they are not owners or publishers and have no responsibility for its content or policies. 


\section{Pacific Journal of Mathematics}

\section{Vol. 14, No. $4 \quad$ August, 1964}

Homer Franklin Bechtell, Jr., Pseudo-Frattini subgroups . . . . . . . . . . . . . . . . 1129

Thomas Kelman Boehme and Andrew Michael Bruckner, Functions with convex means .............................................. 1137

Lutz Bungart, Boundary kernel functions for domains on complex manifolds . . . . . 1151

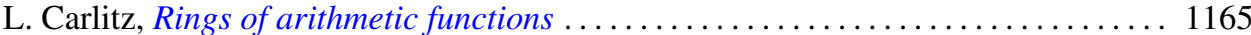

D. S. Carter, Uniqueness of a class of steady plane gravity flows . . . . . . . . . 1173

Richard Albert Dean and Robert Harvey Oehmke, Idempotent semigroups with

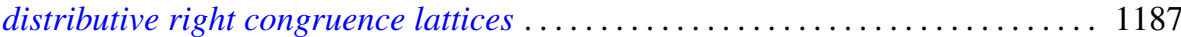

Lester Eli Dubins and David Amiel Freedman, Measurable sets of measures ..... . 1211

Robert Pertsch Gilbert, On class of elliptic partial differential equations in four

variables.......................................... 1223

Harry Gonshor, On abstract affine near-rings .................... 1237

Edward Everett Grace, Cut points in totally non-semi-locally-connected

continua ........................................... 1241

Edward Everett Grace, On local properties and $G_{\delta}$ sets ................ 1245

Keith A. Hardie, A proof of the Nakaoka-Toda formula . . . . . . . . . . . . . . . . 1249

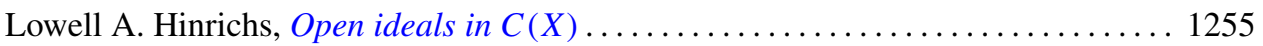

John Rolfe Isbell, Natural sums and abelianizing . . . . . . . . . . . . . . . . 1265

G. W. Kimble, A characterization of extremals for general multiple integral

problems............................................. 1283

Nand Kishore, A representation of the Bernoulli number $B_{n} \ldots \ldots \ldots \ldots \ldots \ldots \ldots \ldots$

Melven Robert Krom, A decision procedure for a class of formulas of first order predicate calculus.

Peter A. Lappan, Identity and uniqueness theorems for automorphic functions ...

Lorraine Doris Lavallee, Mosaics of metric continua and of quasi-Peano spaces . .

Mark Mahowald, On the normal bundle of a manifold..........

J. D. McKnight, Kleene quotient theorems

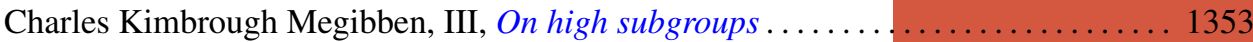

Philip Miles, Derivations on $B^{*}$ algebras . . . . . . . . . . . . . . . . . . . 1359

J. Marshall Osborn, A generalization of power-associativity . . . . . . . . . . . 1367

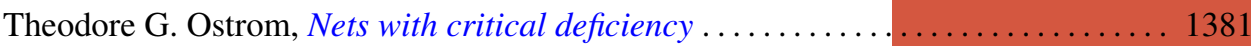

Elvira Rapaport Strasser, On the defining relations of a free product . . . . . . . . . 1389

K. Rogers, A note on orthoganal Latin squares . . . . . . . . . . . . . . . . . . 1395

P. P. Saworotnow, On continuity of multiplication in a complemented algebra ..... 1399

Johanan Schonheim, On coverings . . . . . . . . . . . . . . . . . . . . 1405

Victor Lenard Shapiro, Bounded generalized analytic functions on the torus . . . . . 1413

James D. Stafney, Arens multiplication and convolution . . . . . . . . . . . . . 1423

Daniel Sterling, Coverings of algebraic groups and Lie algebras of classical

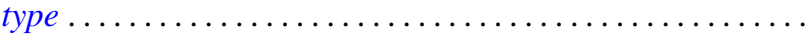

Alfred B. Willcox, Šlov type $C$ algebras over a connected locally compact abelian

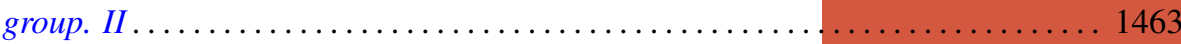

Bertram Yood, Faithful ${ }^{*}$-representations of normed algebras. II . . . . . . . . . 1475

Alexander Zabrodsky, Covering spaces of paracompact spaces 